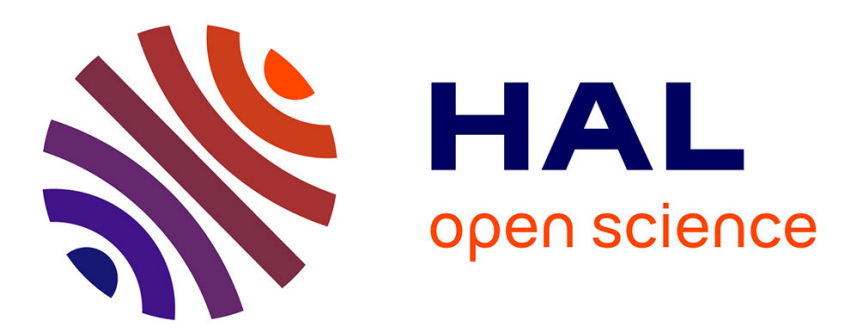

\title{
Implicit Lyapunov-Krasovski Functionals for Time Delay Systems
}

Andrey Polyakov, Denis Efimov, Wilfrid Perruquetti, Jean-Pierre Richard

\section{To cite this version:}

Andrey Polyakov, Denis Efimov, Wilfrid Perruquetti, Jean-Pierre Richard. Implicit LyapunovKrasovski Functionals for Time Delay Systems. Conference on Decision and Control, IEEE, Dec 2014, Los Angeles, United States. hal-01071227

\section{HAL Id: hal-01071227 https://hal.inria.fr/hal-01071227}

Submitted on 3 Oct 2014

HAL is a multi-disciplinary open access archive for the deposit and dissemination of scientific research documents, whether they are published or not. The documents may come from teaching and research institutions in France or abroad, or from public or private research centers.
L'archive ouverte pluridisciplinaire HAL, est destinée au dépôt et à la diffusion de documents scientifiques de niveau recherche, publiés ou non, émanant des établissements d'enseignement et de recherche français ou étrangers, des laboratoires publics ou privés. 


\title{
Implicit Lyapunov-Krasovski Functionals for Time Delay Systems
}

\author{
A. Polyakov, D. Efimov, W. Perruquetti, J.-P. Richard
}

\begin{abstract}
The method of Implicit Lyapunov-Krasovski Functional (ILKF) for stability analysis of time-delay systems is introduced. Theorems on Lyapunov, asymptotic, fiite-time, fixed-time and (hyper-)exponential stability analysis using ILKF are presented. The hyper exponential stabilization algorithm for a time-delay system is developed. The theoretical results are supported by numerical simulations.
\end{abstract}

\section{INTRODUCTION}

The celebrated Second Lyapunov Method was originally founded on the so-called energetic approach to stability analysis. It considers any positive definite function as possible energetic characteristic ("energy") of a dynamic system and the evolution of this "energy" in time is studied. If a dynamic system has an energetic function, which is decreasing (or strictly decreasing) along any trajectory of the system, then this system has some stability property and the corresponding energetic function is called Lyapunov function.

The Lyapunov function method is a main theoretical tool for stability analysis and control synthesis of nonlinear systems ([2], [17]). This method allows us to study the stability properties of the system without finding its solutions. The extension of the Lyapunov's ideas to the case of time delay systems is given by Lyapunov-Razumikhin and LyapunovKrasovski theorems, see, for example, [12], [8], [21], [7], [14]. The corresponding energetic function becomes the functional in the last case. Finding the appropriate Lyapunov function or Lyapunov-Krasovski functional is a difficult problem in the general case.

In addition to asymptotic stability, the control practice also needs to estimate and to adjust the convergence rate. The linear control theory uses the concept of exponential stability for this purpose. The nonlinear control laws may provide much faster (hyper exponential) convergence. A feedback control design providing termination of any transition processes in a finite time (non-asymptotically) is frequently needed for the real applications. The corresponding problem statements appear, for example, in robotic systems, underwater/surface vehicles control systems, aerospace applications, etc. Finite-time control problems are subjects of intensive

Andrey Polyakov, Denis Efimov, Wilfrid Perruquetti are with NonA INRIA - LNE, Parc Scientifique de la Haute Borne 40, avenue Halley Bat.A, Park Plaza 59650 Villeneuve d'Ascq, France (e-mail: andrey.polyakov@inria.fr, denis.efimov@inria.fr, wilfrid.perruetti@inria.fr, jean-pierre.richard@ec-lille.fr). They are also with LAGIS UMR CNRS 8219, Ecole Centrale de Lille, BP 48, Cité Scientifique, 59651 Villeneuved'Ascq, France. D. Efimov is also with Department of Control Systems and Informatics, Saint Petersburg State University of Information Technologies Mechanics and Optics (ITMO), 49 Kronverkskiy av., 197101 Saint Petersburg, Russia. This work was supported in part by the Government of Russian Federation (Grant 074-U01) and the Ministry of Education and Science of Russian Federation (Project 14.Z50.31.0031) researches in the last years; e.g., see [9], [3], [11], [16], [17], [18]. The method of Lyapunov (-Krasovski) functions helps to estimate the rate of convergence and to adjust the control parameters in order to provide the desired rate.

Recently, the Implicit Lyapunov Function(ILF) method has been developed for non-asymptotic (finite-time and fixedtime) analysis, see [19]. This method allows us to design the control together with the Lyapunov function for the closedloop system. A simple procedure for parameter tuning based on Linear Matrix Inequalities (LMIs) was developed for linear plants.

The ideas, which underlines the ILF method, was initially introduced by [13] for control synthesis problems. The corresponding design methodology was called controllability function method. In order to be more precise we follow more recent terminology of [1]. The ILF method uses Lyapunov function defined in the implicit form by some algebraic equations. Stability analysis in this case does not require solving of this equation, since the Implicit function theorem (see, for example, [5]) helps to check all stability conditions by analyzing the algebraic equation directly.

This paper extends the concept of ILF to the case of time-delay systems. The stability theorems presented in the paper introduce the ILKF method for Lyapunov, asymptotic, (hyper) exponential and non-asymptotic (finite-time and fixed-time) stability analysis of time-delay systems. They allow us to define the Lyapunov-Krasovski functionals in the implicit form. The developed method is demonstrated on the control design problem for hyper exponential stabilization of a special time-delay linear system. The procedure for design of control parameters is presented in the LMI form.

The paper is organized as follows. The next section introduces some notations used in the paper. Then the method of ILKF is presented. Next, the problem of hyper exponential stabilization of the chain of integrators with delay-depended terms is studied in order to demonstrate the ILKF-based control approach. Finally, the numerical example and conclusions are presented.

\section{NOTATION}

Through the paper the following notation will be used:

- $\mathbb{R}_{+}=\{x \in \mathbb{R}: x>0\}$, where $\mathbb{R}$ is the set of real number;

- $\mathbb{N}$ is the set of nonnegative integers;

- $\|\cdot\|$ is the Euclidian norm in $\mathbb{R}^{n}$, i.e. $\|x\|=$ $\sqrt{x_{1}^{2}+\ldots+x_{n}^{2}}$ for $x=\left(x_{1}, \ldots, x_{n}\right)^{T} \in \mathbb{R}^{n}$;

- $\operatorname{diag}\left\{\lambda_{i}\right\}_{i=1}^{n}$ is the diagonal matrix with the elements $\lambda_{i}$ on the main diagonal; 
- a continuous function $\sigma: \mathbb{R}_{+} \rightarrow \mathbb{R}_{+}$belongs to the class $\mathcal{K}$ if it is strictly increasing and $\sigma\left(0^{+}\right)=0^{+}$; if additionally it is radially unbounded then $\sigma$ belongs to $\mathcal{K}_{\infty}$

- for a matrix $P \in \mathbb{R}^{n \times n}$ which has the real spectrum, the minimal and maximal eigenvalues are denoted by $\lambda_{\min }(P)$ and $\lambda_{\max }(P)$, respectively;

- if $P \in \mathbb{R}^{n \times n}$ then the inequality $P>0(P \geq 0$, $P<0, P \leq 0)$ means that $P$ is symmetric and positive definite (positive semidefinite, negative definite, negative semidefinite).

- For $P>0$ the matrix $P^{1 / 2}:=B$ is such that $B^{2}=P$.

- $\mathbb{C}_{[a, b]}\left(\mathbb{R}^{n}\right)$ is the set of continuous functions $\mathbb{R} \rightarrow \mathbb{R}^{n}$ defined on $[a, b]$.

- Let $\mathbb{C}_{h}=\mathbb{C}_{[-h, 0]}\left(\mathbb{R}^{n}\right)$ and $\|\cdot\|_{h}$ is the norm in $\mathbb{C}_{h}$ defined as follows $\|\varphi\|_{h}=\max _{t \in[-h, 0]}\|\varphi(t)\|$ for $\varphi \in \mathbb{C}_{h}$.

\section{Stability Definitions}

Consider the system of the form

$$
\begin{aligned}
& \dot{x}(t)=f\left(t, x_{h}(t)\right), \quad t \in \mathbb{R}_{+} \\
& x(t)=\varphi(t) \in \mathbb{C}_{h}, \quad t \in[-h, 0]
\end{aligned}
$$

where $x \in \mathbb{R}^{n}, x_{h}(t) \in \mathbb{C}_{h}$ is the state function defined by means of the formula $x_{h}(t):=x(t+\tau)$ with $\tau \in[-h, 0]$, $h \in \mathbb{R}_{+}$is delay and $f: \mathbb{R}_{+} \times \mathbb{C}_{h} \rightarrow \mathbb{R}^{n}$ is a continuous operator.

Assume that the origin is an equilibrium point of the system (1), i.e. $f(t, 0)=0$.

A solution of the system (1) with the given initial function $\varphi \in \mathbb{C}_{h}$ is denoted by $x(t, \varphi)$.

Definition 1 ([10]): The origin of the system (1) is said to be Lyapunov stable if there exists $\delta>0$ and a function $\varepsilon \in \mathcal{K}$ such that $\forall \varphi \in \mathbb{C}_{h}:\|\varphi\|_{h}<\delta$ and $\forall x(t, \varphi)$ the following inequality

$$
\|x(t, \varphi)\| \leq \varepsilon\left(\|\varphi\|_{h}\right), \quad t \in \mathbb{R}_{+},
$$

holds.

Definition 2 ([10]): The origin of the system (1) is said to be asymptotically stable, if it is Lyapunov stable and $\forall \varphi \in$ $\mathbb{C}_{h}:\|\varphi\|_{h}<\delta$ and $\forall x(t, \varphi)$ the following property

$$
\lim _{t \rightarrow+\infty}\|x(t, \varphi)\|=0
$$

holds. If $\delta=+\infty$ then the origin of the system (1) is said to be globally asymptotically stable.

The stability definitions 1 and 2 investigate only qualitative properties of the system.

Definition 3 ([10]): The origin of the system (1) is said to be exponentially stable, if it is asymptotically stable and $\forall \varphi \in \mathbb{C}_{h}:\|\varphi\|_{h}<\delta$ and $\forall x(t, \varphi)$ the following property

$$
\|x(t, \varphi)\| \leq \sigma\left(\|\varphi\|_{h}\right) e^{-\alpha t}, \quad t \in \mathbb{R}_{+}
$$

holds for some $\sigma \in \mathcal{K}_{\infty}$ and for some $\alpha \in \mathbb{R}_{+}$. If $\delta=$ $+\infty$ then the origin of the system (1) is said to be globally exponentially stable.

The parameter $\alpha \in \mathbb{R}_{+}$characterizes the rate of exponential convergence.
Let us introduce the functions $\rho_{r}: \mathbb{R} \rightarrow \mathbb{R}$ by the following recursive formula

$$
\rho_{0}(z)=z, \quad \rho_{r}(z)=e^{\rho_{r-1}(z)},
$$

where $r \geq 1$. The next definition introduces the novel asymptotic stability property with faster convergence rate than any exponential. It follows the ideas of hyperpower (see, [15]).

Definition 4: The origin of the system (1) is said to be hyper exponentially stable of degree $r \geq 1$, if it is asymptotically stable and $\forall \varphi \in \mathbb{C}_{h}:\|\varphi\|_{h}<\delta, \forall x(t, \varphi)$ the following property

$$
\|x(t, \varphi)\| \leq \sigma\left(\|\varphi\|_{h}\right) e^{-\rho_{r}(\alpha t)}, \quad t \in \mathbb{R}_{+}
$$

holds for some $\sigma \in \mathcal{K}_{\infty}$ and for some $\alpha \in \mathbb{R}_{+}$. If $\delta=+\infty$ then the origin of the system (1) is said to be globally hyper exponentially stable.

By analogy with exponential stability we say that the number $\alpha$ from the last definition defines the rate of hyper exponential convergence.

For example, the following delay-free system

$$
\dot{x}=-(1-\ln (|x(t)|)) x(t), \quad x \in \mathbb{R}
$$

is locally hyper exponentially stable with the rate $\alpha=1$ and degree $k=1$.

The next two definitions present the non-asymptotic stability.

Definition 5 ([16]): The origin of the system (1) is said to be globally finite-time stable, if it is Lyapunov stable and finite-time attractive, i.e. $T: \mathbb{C}_{[-h, 0]} \rightarrow \mathbb{R}_{+}$, such that for $\forall \varphi \in \mathbb{C}_{h}:\|\varphi\|_{h}<\delta$ and $\forall x(t, \varphi)$ the following property

$$
\lim _{t \rightarrow T(\varphi)}\|x(t, \varphi)\|=0
$$

holds. If $\delta=+\infty$ then the origin of the system (1) is said to be globally finite-time stable.

The functional $T(\cdot)$ from Definition 5 is called the settlingtime functional of the system (1).

Examples of finite-time stable systems of the form (1) are given in [16].

The next definition extends the concept of fixed-time stability by [18] to the case of time-delay systems.

Definition 6: The origin of the system (1) is said to be globally fixed-time stable, if it is finite-time stable and the settling-time functional $T: \mathbb{C}_{[-h, 0]} \rightarrow \mathbb{R}_{+}$is bounded, i.e. there exists $T_{\max } \in \mathbb{R}_{+}$such that

$$
\sup _{\varphi \in \mathbb{C}_{h}:\|\varphi\|_{h}<\delta} T(\varphi)=T_{\max }
$$

for some $\delta \in \mathbb{R}_{+}$. If $\delta=+\infty$ then the origin of the system (1) is said to be globally fixed-time stable.

\section{Stability Analysis of Time-Delay Systems USING IMPLICIT LYAPUNOV-KRASOVSKI FUNCTIONAL}

\section{A. Implicit functions and operators}

The next lemma provides simple characterization of the implicit functions belonging to the class $\mathcal{K}_{\infty}$. 
Lemma 1: If the function

$$
\begin{gathered}
q: \mathbb{R}_{+}^{2} \rightarrow \mathbb{R}, \\
(\sigma, s) \rightarrow q(\sigma, s)
\end{gathered}
$$

is such that $\mathbb{R}_{+}^{2}$

1) $q$ is continuously differentiable in both arguments on

2) for any $s \in \mathbb{R}_{+}$there exists $\sigma^{-}, \sigma^{+} \in \mathbb{R}_{+}$such that

$$
q\left(\sigma^{-}, s\right)<0<q\left(\sigma^{+}, s\right)
$$

3) the inequalities

$$
\frac{\partial q}{\partial \sigma}<0 \text { and } \frac{\partial q}{\partial s}>0
$$

hold for $(\sigma, s) \in \mathbb{R}_{+}^{2}$;

4) for $\Gamma=\left\{(\sigma, s) \in \mathbb{R}_{+}^{2}: q(\sigma, s)=0\right\}$

$$
\lim _{\substack{s \rightarrow 0^{+} \\(\sigma, s) \in \Gamma}} \sigma=0^{+}, \lim _{\substack{\sigma \rightarrow 0^{+} \\(\sigma, s) \in \Gamma}} s=0^{+}, \lim _{\substack{s \rightarrow+\infty \\(\sigma, s) \in \Gamma}} \sigma=+\infty ;
$$

then the implicit function $\sigma$ defined by the equation $q(\sigma, s)=$ 0 is of the class $\mathcal{K}_{\infty}$.

All proofs are omitted in order to satisfy the space restriction.

By definition, a function $q: \mathbb{R}_{+}^{2} \rightarrow \mathbb{R}_{+}$belongs to the class $\mathcal{I} \mathcal{K}_{\infty}$ if it satisfies the conditions of Lemma 1.

In order to present the implicit function theorem in a Banach Spaces let us recall some standard definitions.

The operator $g: \mathbb{Z} \rightarrow \mathbb{Y}$, where $\mathbb{Z}$ and $\mathbb{Y}$ are Banach spaces, is called F-differentiable (Frèchet differentiable) at the point $z_{0} \in \mathbb{Z}$ if there exists a linear operator $D g_{z_{0}}: \mathbb{Z} \rightarrow$ $\mathbb{Y}$ such that

$$
\frac{\left\|g(z)-g\left(z_{0}\right)-D g_{z_{0}}\left(z-z_{0}\right)\right\|_{\mathbb{Y}}}{\left\|z-z_{0}\right\|_{\mathbb{Z}}} \rightarrow 0 \quad \text { as }\left\|z-z_{0}\right\| \rightarrow 0
$$

where $\|\cdot\|_{\mathbb{Z}}$ and $\|\cdot\|_{\mathbb{Y}}$ are norms in the Banach spaces $\mathbb{Z}$ and $\mathbb{Y}$, respectively.

The operator $p: \mathbb{Z} \rightarrow \mathbb{Y}$ is continuous at the point $z_{0} \in \mathbb{Z}$ if there exists a function $\sigma \in \mathcal{K}$ such that $\left\|p(z)-p\left(z_{0}\right)\right\|_{\mathbb{Z}} \leq$ $\sigma\left(\left\|z-z_{0}\right\|_{\mathbb{Y}}\right)$ for any $z \in B\left(z_{0}\right) \subset \mathbb{Z}$, where $B\left(z_{0}\right)$ is some ball with the center at $z_{0}$.

Theorem 1 ([6]): Let $\mathbb{Z}, \mathbb{Y}$ and $\mathbb{Q}$ be Banach spaces. Let $g: \mathbb{Z} \times \mathbb{Y} \rightarrow \mathbb{Q}$ be a continuous operator such that

1) $g\left(z_{0}, y_{0}\right)=0$ for some $z_{0} \in \mathbb{Z}$ and $y_{0} \in \mathbb{Y}$;

2) $g$ is F-differentiable in the neighborhood of the point $\left(z_{0}, y_{0}\right)$

3) $D g_{z_{0}}^{\prime}$ is invertible, where $g^{\prime}(z):=g\left(z, y_{0}\right)$.

Then there exists a unique operator $f: \mathbb{Y} \rightarrow \mathbb{Z}$ defined in an open neighborhood $U_{0}$ of $y_{0}$ such that $g(f(y), y)=0$ for all $y \in U_{0}$. Moreover, if $g$ is continuously F-differentiable at a neighborhood of $\left(z_{0}, y_{0}\right)$ then $f$ is also continuously differentiable at some neighborhood of $y_{0}$.

\section{B. Theorems on Implicit Lyapunov-Krasovski Functional}

The next theorem shows that the stability analysis of time delay systems can be provided using Implicit LyapunovKrasovski Functionals.

Theorem 2: If there exists a continuous functional

$$
\begin{gathered}
Q: \mathbb{R}_{+} \times \mathbb{C}_{[-h, 0]} \rightarrow \mathbb{R} \\
(V, y) \mapsto Q(V, y)
\end{gathered}
$$

such that:

C1) $Q$ is continuously F-differentiable on $\mathbb{R}_{+} \times \mathbb{C}_{[-h, 0]}$;

C2) the inequality

$$
\frac{\partial Q(V, y)}{\partial V}<0
$$

holds for $\forall V \in \mathbb{R}_{+}$and $\forall y \in \mathbb{C}_{h} \backslash\{0\}$;

C3) there exist the implicit functions $q_{i} \in \mathcal{I} \mathcal{K}_{\infty}, i=1,2$, such that

$$
q_{1}(V,\|y(0)\|) \leq Q(V, y(\cdot)) \leq q_{2}\left(V,\|y\|_{h}\right),
$$

for all $V \in \mathbb{R}_{+}$and all $y \in \mathbb{C}_{[-h, 0]}$;

C4) $\forall\left(V, x_{h}(t)\right) \in \Omega$ such that $x(t)$ satisfies (1) we have

$$
\frac{\partial Q\left(V, x_{h}(t)\right)}{\partial t} \leq-\sigma(\|x(t)\|), \quad \forall t \in \mathbb{R}_{+},
$$

where

$$
\Omega=\left\{(V, y) \in \mathbb{R}_{+} \times \mathbb{C}_{h}: Q(V, y)=0\right\}
$$

and $\sigma: \mathbb{R} \rightarrow \mathbb{R}$. Then the origin of the system (1) is

- Lyapunov stable if $\sigma$ is a nonnegative function;

- asymptotically stable if $\sigma \in \mathcal{K}$.

It is worth to stress that, similarly to the classical method of Lyapunov-Krasovski functionals, the condition C4) can be checked using the right-hand side of the equation (1) only (see, for example, the next section).

The simple modification of the last theorem provides a tool for global asymptotic and finite-time stability analysis of time-delay systems.

Theorem 3: Let there exists a continuous functional

$$
\begin{gathered}
Q: \mathbb{R}_{+} \times \mathbb{C}_{h} \rightarrow \mathbb{R}, \\
(V, y) \mapsto Q(V, y)),
\end{gathered}
$$

which satisfies the conditions $C 1)-C 3$ ) and the condition:

C4bis) $\forall t \in \mathbb{R}_{+}$and $\forall\left(V, x_{h}(t)\right) \in \Omega: 0<V \leq V^{\max }$ such that $x_{h}(t)$ satisfies (1) we have

$$
\frac{\partial Q\left(V, x_{h}(t)\right)}{\partial t} \leq \sigma(V) \frac{\partial Q\left(V, x_{h}(t)\right)}{\partial V}, \quad \forall t \in \mathbb{R}_{+},
$$

where $\Omega$ is defined by (2).

- If $\sigma \in \mathcal{K}_{\infty}$ and $V^{\max }=+\infty$ then the origin of system (1) is globally asymptotically stable;

- If $\sigma(V):=\alpha V^{1-\mu}, \alpha, V^{\max } \in \mathbb{R}_{+}$and $\mu \in(0,1]$ then the origin of system (1) is finite-time stable (globally finite-time stable for $V^{\max }=+\infty$ ) with the following settling time estimate:

$$
T(\varphi) \leq \frac{V_{0}^{\mu}}{\alpha \mu}
$$

where $V_{0} \in \mathbb{R}_{+}: Q\left(V_{0}, \varphi\right)=0$.

Proof of this theorem follows from Theorem 2 and Proposition 4 presented in [16]. Indeed, the condition C4bis) guarantees $\dot{V} \leq-\alpha V^{1-\mu}$.

Theorem 4: If there exist two continuous functionals

$$
\begin{array}{cc}
Q_{1}: \mathbb{R}_{+} \times \mathbb{C}_{h} \rightarrow \mathbb{R}, & Q_{2}: \mathbb{R}_{+} \times \mathbb{C}_{h} \rightarrow \mathbb{R}, \\
(V, y) \mapsto Q_{1}(V, y), & (V, y) \mapsto Q_{2}(V, y),
\end{array}
$$


which satisfy the conditions $C 1$ )-C3) of Theorem 2, the functional $Q_{1}$ satisfies the condition $C 4 b i s$ ) of Theorem 3 with $V^{\max }=1$, and in addition

C5) $Q_{1}(1, y)=Q_{2}(1, y)$ for all $y \in \mathbb{C}_{h}:\|y\|<\delta$, where $\delta \in \mathbb{R}_{+}$is some constant;

C6) for $\forall t \in \mathbb{R}_{+}$and $\forall\left(V, x_{h}(t)\right) \in \Omega$ and $V \geq 1$ such that $x_{h}(t)$ satisfies (1) we have

$$
\frac{\partial Q_{2}\left(V, x_{h}(t)\right)}{\partial t} \leq \beta V^{1+\nu} \frac{\partial Q\left(V, x_{h}(t)\right)}{\partial V}, \quad \forall t \in \mathbb{R}_{+}
$$

where $\beta, \nu \in \mathbb{R}_{+}$are some constants,

then the origin of system (1) is fixed-time stable (globally fixed-time stable if $\delta=+\infty$ ) with the following settling time estimate:

$$
T(\varphi) \leq \frac{1}{\alpha \mu}+\frac{1}{\beta \nu}
$$

Implicit Lyapunov-Krasovski functional-based analysis of exponential and hyper exponential stability requires some additional restrictions to the class of functionals to be used.

Theorem 5: If there exists a continuous functional

$$
\begin{gathered}
Q: \mathbb{R}_{+} \times \mathbb{C}_{h} \rightarrow \mathbb{R}, \\
(V, y) \mapsto Q(V, y),
\end{gathered}
$$

which satisfies the conditions $C 1)-C 3$ ) and the conditions

C3*) there exists $c \in \mathbb{R}_{+}$such that

$$
\frac{\partial q_{1}}{\partial s}+c \frac{\partial q_{1}}{\partial \sigma} \geq 0
$$

for all $(\sigma, s) \in \mathbb{R}^{2}$ such that $q_{1}(\sigma, s)=0$;

$\left.C 4^{*}\right)$ for $\forall t \in \mathbb{R}_{+}$and $\forall\left(V, x_{h}(t)\right) \in \Omega$ and $0<V \leq$ $V^{\max }$ such that $x(t)$ satisfies (1) we have

$$
\frac{\partial Q\left(V, x_{h}(t)\right)}{\partial t} \leq \alpha p(V) V \frac{\partial Q\left(V, x_{h}(t)\right)}{\partial V}, \quad \forall t \in \mathbb{R}_{+},
$$

where $\alpha, V^{\max } \in \mathbb{R}_{+}$are some constants, then the origin of system (1) is

- exponentially stable if $p(V)=1$;

- hyper exponentially stable with degree $r=1$ if

$$
p(V)=\left\{\begin{array}{lll}
1+\ln (V) & \text { for } \quad V>1 \\
1-\ln (V) & \text { for } \quad V \leq 1
\end{array}\right.
$$

with the rate of convergence $\alpha$. The global (hyper) exponential stability is provided if $V^{\max }=+\infty$.

\section{EXAMPLE: HyPER EXPONENTIAL StABILIZATION}

\section{A. Problem formulation}

Let us consider a single input control system of the form

$$
\left\{\begin{array}{l}
\dot{x}(t)=A_{0} x(t)+A_{1} x(t-h)+b u(t), \quad t>0, \\
x(t)=\varphi(t) \in \mathbb{C}, \quad t \in[-h, 0],
\end{array}\right.
$$

where $x \in \mathbb{R}^{n}$ is the state vector, $u \in \mathbb{R}$ is a control input, $A_{0}, A_{1} \in \mathbb{R}^{n \times n}$ are the system matrices of the form

$A_{0}=\left(\begin{array}{cccc}0 & 1 & \ldots & 0 \\ \ldots & \ldots & \ldots & \ldots \\ 0 & 0 & \ldots & 1 \\ 0 & 0 & \ldots & 0\end{array}\right), A_{1}=\left(\begin{array}{cccc}a_{1} & 0 & \ldots & 0 \\ \ldots & \ldots & \ldots & \ldots \\ 0 & 0 & \ldots & 0 \\ 0 & 0 & \ldots & a_{n}\end{array}\right)$,

and the vector $b \in \mathbb{R}^{n \times 1}$ is of the form $b=(0,0, \ldots, 1)^{\top}$.

The problem is to design a control algorithm, which hyper exponentially stabilizes the system (5) at the origin.

\section{B. Hyper Exponential Control Design}

Introduce the Implicit Lyapunov-Krasovski Functional (ILKF) of the form

$$
\begin{gathered}
Q(V, y(\cdot)):=-1+y^{\top}(0) D\left(V^{-1}\right) P D\left(V^{-1}\right) y(0)+ \\
\quad \int_{-h}^{0}\left(\frac{e}{V}\right)^{\frac{2 \tau+h}{h}} y^{\top}(\tau) D\left(V^{-1}\right) S D\left(V^{-1}\right) y(\tau) d \tau,
\end{gathered}
$$

where $D(\lambda)$ is the dilation matrix of the form

$$
D(\lambda)=\operatorname{diag}\left\{\lambda^{1+(n-i)}\right\}_{i=1}^{n},
$$

and $P, S \in \mathbb{R}^{n \times n}$ are symmetric positive definite matrices. Denote $H:=\operatorname{diag}\{1+(n-i)\}_{i=1}^{n}$.

The next theorem presents the scheme of the ILKF-based control design.

Theorem 6: Let $X \in \mathbb{R}^{n \times n}, Z \in \mathbb{R}^{n \times n}$ and $y \in \mathbb{R}^{1 \times n}$ satisfy the following system of LMIs:

$$
\left.\begin{array}{c}
A_{0} X+X A_{0}^{\top}+b y+y^{\top} b^{\top}+\quad X \\
\frac{2}{h} X+e A_{1} Z A_{1}^{\top} \\
X \quad \frac{-1}{e} Z
\end{array}\right) \leq 0,
$$

Then the control of the form

$$
u(V, x(t))=k D\left(V^{-1}\right) x(t),
$$

where $k=y X^{-1}, V \in \mathbb{R}_{+}: Q\left(V, x_{h}(t)\right)=0$ and $Q(V, y)$ is defined by (6) with $P=X^{-1}$ and $S=Z^{-1}$, stabilizes the origin of the system (5) hyper exponentially with degree $r=1$, the domain of initial conditions

$$
\Omega=\{\varphi \in \mathbb{C}: Q(1, \varphi) \leq 0\} .
$$

and the convergence rate $\frac{2}{\gamma h}$, where

$$
\gamma=\max \left\{\lambda_{\max }\left(X^{\frac{1}{2}} H+H X^{\frac{1}{2}}\right), 1+\lambda_{\max }\left(Z^{\frac{1}{2}} H+H Z^{\frac{1}{2}}\right)\right\} .
$$

The advantage of the presented control scheme is the LMI representation of the procedure for parameters tuning. Any existing LMI solver can be used in order to select the control parameters.

\section{Practical implementation}

In order to apply the control (8) in practice it is necessary to present the procedure for calculation of the value $V$ for any given $y(\cdot) \in \mathbb{C}_{h}$ such that $Q(V, y(\cdot))=0$. Since $Q$ satisfies the condition $C 2$ ) of Theorem 2 then for any $y(\cdot)$ the ILF equation has a unique solution, which can be easily found by means of, for example, bisection method.

The alternative approach to realization of the ILKF-based control scheme is adaptive. For example, we can realize the Newton method for numerical calculation of the solution of the equation $Q(V, y(\cdot))=0$. The simplest continuous-time algorithm is the following

$$
\dot{V}_{n}=-p Q\left(V_{n}, y(\cdot)\right)\left[\frac{\partial Q\left(V_{n}, y(\cdot)\right)}{\partial V_{n}}\right]^{-1}, \quad p \in \mathbb{R}_{+} .
$$

For any fixed $y(\cdot)$ the presented gradient method guarantees $V_{n} \rightarrow V: Q(V, y(\cdot))=0$. In order to boost the convergence 
of Newton method its fast modification can be considered (see, for example, [4]).

Therefore, the ILKF control can be realized in a sufficiently fast digital control device. Below we presents the results A detailed study of the practical application of the ILKF control algorithms goes out of the scope of this paper.

\section{Numerical Simulation}

Consider the problem of hyper exponential stabilization of the system (5) with $n=2$, the matrix $A_{1}=\operatorname{diag}\{0.1,0\}$ and the delay $h=0.5$. By solving of the LMI system (7) we obtain

$$
\begin{gathered}
P=\left(\begin{array}{cc}
11.0538 & 1.6821 \\
1.6821 & 0.3494
\end{array}\right), \quad S=\left(\begin{array}{ll}
1.9418 & 0.0880 \\
0.0880 & 0.0488
\end{array}\right), \\
K=\left(\begin{array}{ll}
-96.2124 & -17.0123
\end{array}\right) \text { and } \gamma=14.4407 .
\end{gathered}
$$

The simulations for the ILKF control application have been made in MATLAB Simulink. The value of ILKF was calculated on-line using the Newton method (9) with $p=$ $10^{3}$. All integrals, which appear in (9), were approximated by Simpson formula. In order to avoid singularities during calculation of $Q(V, y(\cdot))$ the admissible value of $V$ is also bounded from below by $V_{\min }=0.04$. The parameter $V_{\min }$ have been selected manually. The development of the procedure for its selection goes out of the scope of this paper.

In order to demonstrate the robustness, the simulations of the closed-loop system has been perturbed by the additive matched exogenous disturbance $d(t)=10 b \sin (10 t)$ so that the term $b u$ is replaced with $b(u+10 \sin (10 t))$ in (5).

The results of simulations are depicted on Fig. 2-4. They demonstrate the high control precision and under disturbance rejection similar to sliding mode control algorithms. Indeed, the control signal tends to the equivalent control $u_{e q}=$ $-10 \sin (10 t)$ (see, Fig. 3).

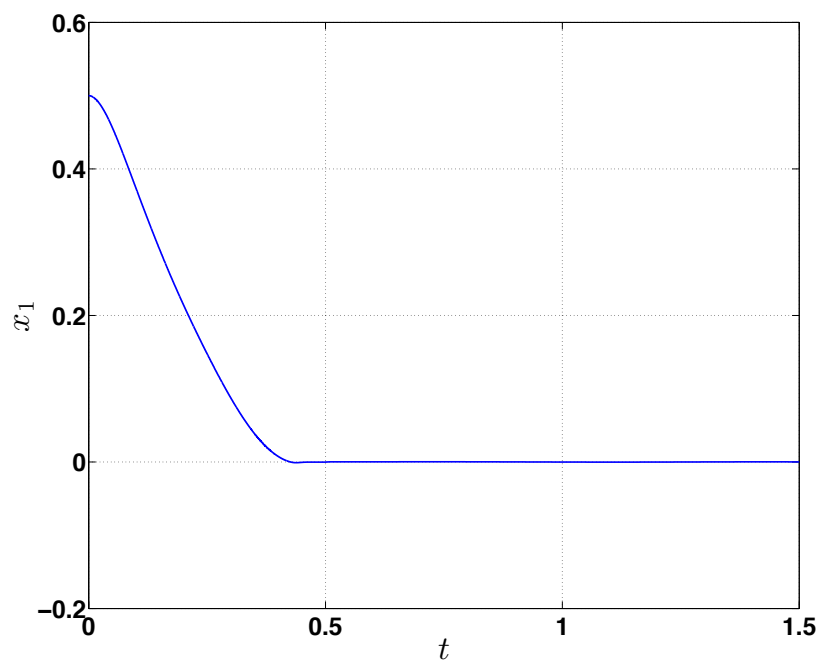

Fig. 1. Evolution of $x_{1}$ coordinate.

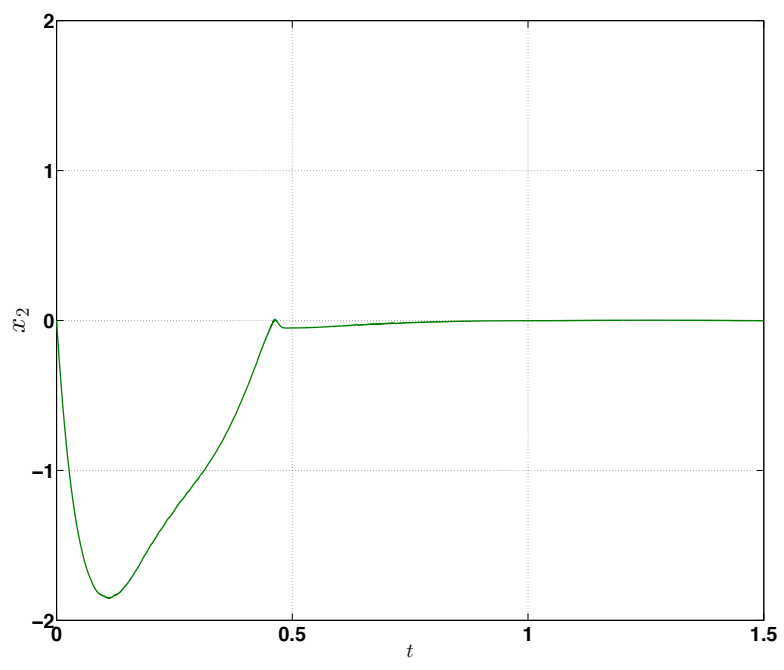

Fig. 2. Evolution of $x_{2}$ coordinate.

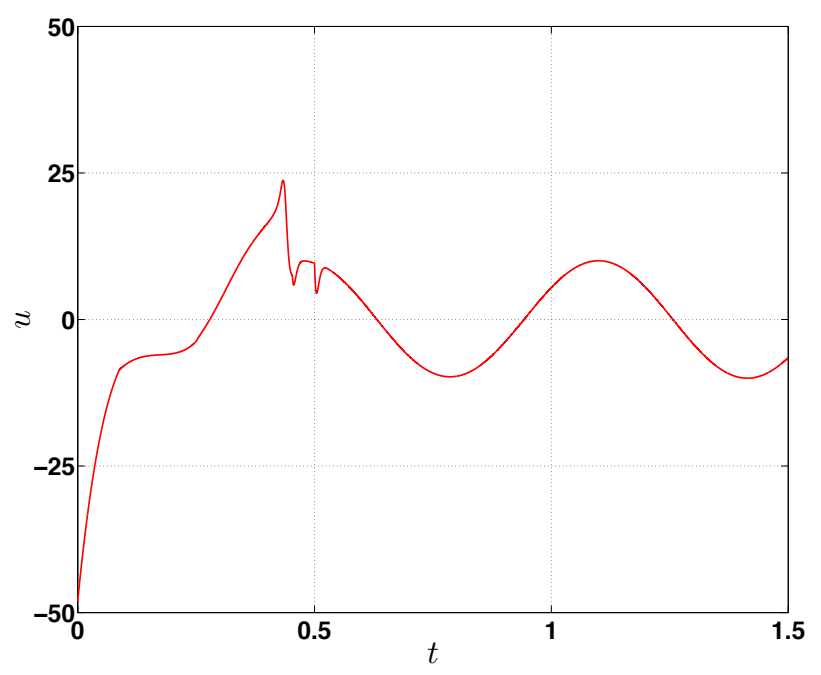

Fig. 3. The control input.

\section{CONCLUSIONS}

The paper presents the method of the implicit LyapunovKrasovski functionals. Theorems on Lyapunov-Krasovski functionals for Lyapunov, asymptotic, finite-time, fixed-time and (hyper)-exponential stability analysis are presented. The conditions of these theorems are more complicated than classical ones. For instance, they uses implicit functions of the class $K_{\infty}$. However, the implicit approach allows us to design fast control laws for time-delay systems. Based on the simple modification classical Lyapunov-Krasovski functional the hyper-exponential stabilizing feedback has been designed for a state-delay liner system. ILFK method can be also applied for observer design (like in [20]). This problem is the topic for possible future research. 


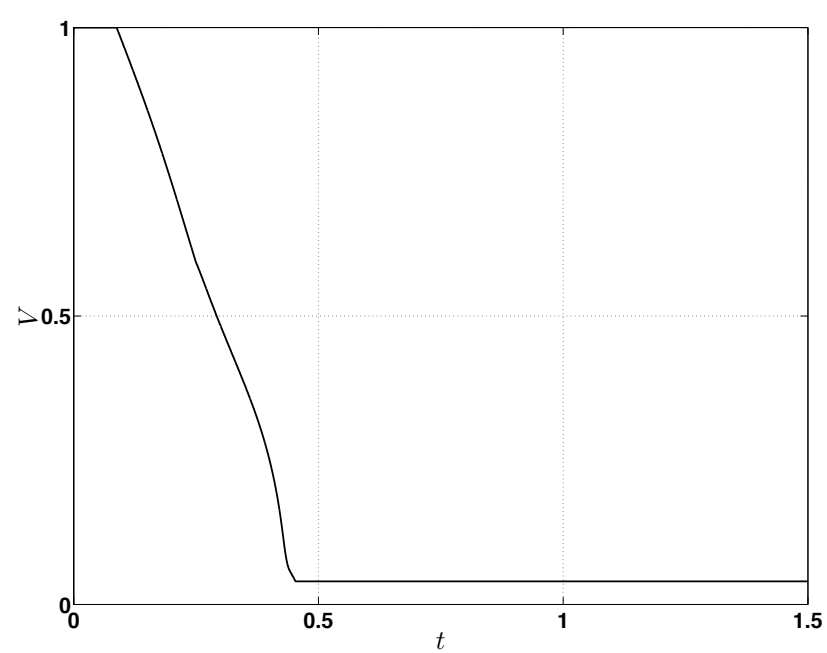

Fig. 4. The evolution of ILKF.

\section{REFERENCES}

[1] J. Adamy and A. Flemming. Soft variable-structure controls: a survey. Automatica, 40:1821-1844, 2004.

[2] A. Bacciotti and L. Rosier. Lyapunov Functions and Stability in Control Theory. Springer, 2nd edition, 2005.

[3] S.P. Bhat and D.S. Bernstein. Finite-time stability of continuous autonomous systems. SIAM Journal of Control and Optimization, 38(3):751-766, 2000.

[4] J. Cortes. Finite-time convergent gradient flows with applications to network consensus. Automatica, 42(11):1993-2000, 2006.

[5] R. Courant and F. John. Introduction to calculus and analysis (Vol. II/1). New York: Springer, 2000.

[6] B.K. Driver. Analysis Tools with Applications. Springer, 2003.

[7] E. Fridman. Descriptor discretized lyapunov functional method: Analysis and design. IEEE Transactions on Automatic Control, 51(5):890-897, 2006.

[8] K. Gu, V. Kharitonov, and J. Chen. Stability of Time-Delay Systems. Boston, MA: Birkhauser, 2003.

[9] V.T. Haimo. Finite time controllers. SIAM Journal of Control and Optimization, 24(4):760-770, 1986.

[10] J. Hale. Theory of Functional Differential Equations. Springer-Verlag, 1977.

[11] Y. Hong. Finite-time stabilization and stabilizability of a class of controllable systems. Systems \& Control Letters, 46(4):231-236, 2002.

[12] V.L. Kharitonov. Robust stability analysis of time delay systems: A survey. Annual Review in Control, 23:185-196, 1999.

[13] V.I. Korobov. A general approach to synthesis problem. Doklady Academii Nauk SSSR, 248:1051-1063, 1979.

[14] M. Krstic. Delay Compensation for Nonlinear, Adaptive, and PDE Systems. Birkhauser Boston, 2009.

[15] J. Macdonnell. Some critical points on the hyperpower function ${ }^{n} x=$ $x^{x^{x}}$. International Journal of Mathematical Eduction in Science and Technology, 20(2):297-305, 1989.

[16] E. Moulay, M. Dambrine, N. Yeganefar, and W. Perruquetti. Finitetime stability and stabilization of time-delay systems. System \& Control Letters, 57(7):561-566, 2008.

[17] Y. Orlov. Discontinous systems: Lyapunov analysis and robust synthesis under uncertainty conditions. Springer-Verlag, 2009.

[18] A. Polyakov. Nonlinear feedback design for fixed-time stabilization of linear control systems. IEEE Transactions on Automatic Control, 57(8):2106-2110, 2012.

[19] A. Polyakov, D. Efimov, and W. Perruquetti. Finite-time stabilization using implicit lyapunov function technique. In 9th Symposium on Nonlinear Control Systems, pages 140-145, 2013.

[20] A. Polyakov, D. Efimov, and W. Perruquetti. Homogeneous differentiator design using implicit lyapunov function method. In European Control Conference, pages 288-293, 2014.
[21] J.-P. Richard. Time-delay systems: An overview of some recent advances and open problems. Automatica, 39(10):1667-1694, 2003. 